\title{
Newtonyen Olmayan Lebesgue Ölçüsü
}

\author{
Non-Newtonian Lebesgue Measure
}

\section{Oğuz OĞUR*1,a, Sezgin DEMİR ${ }^{2, b}$}

${ }^{1}$ Giresun Üniversitesi, Eğitim Fakültesi, Matematik ve Fen Bilimleri Eğitimi Bölümü, 28200, Giresun ${ }^{2}$ Giresun Üniversitesi, Fen-Edebiyat Fakültesi, Matematik Bölümü, 28200, Giresun

• Geliş tarihi / Received: 30.07 .2019 •Düzeltilerek geliş tarihi / Received in revised form: 08.10.2019 • Kabul tarihi / Accepted: 23.10 .2019

\section{$\ddot{\mathbf{O z}}$}

Bu çalışmada, Newtonyen olmayan sınırlı kümelerin iç ve dış ölçü kavramları verildi. Ayrıca, Newtonyen olmayan kümelerin Lebesgue anlamında ölçüsü tanımlanarak ilgili temel teoremler verildi.

Anahtar kelimeler: Newtonyen Olmayan Dış Ölçü, Newtonyen Olmayan İç Ölçü, Newtonyen Olmayan Ölçü

\begin{abstract}
In this paper, definitions of the inner and outer measure of non-Newtonian bounded sets are given. Also, Lebesgue measure of non-Newtonian sets is defined and related theorems are given.
\end{abstract}

Keywords: Non-Newtonian Outer Measure, Non-Newtonian Inner Measure, Non-Newtonian Measure 


\section{Giriş}

Newtonyen olmayan kalkülüs 1967-1970 yılları arasında Michael Grossman ve Robert Katz tarafindan oluşturulmuştur. Önce klasik kalkülüsü daha sonra sirasıyla Geometrik, Harmonik ve Kuadratik kalkülüsü oluşturmuşlardır (Grossman ve Katz, 1972).

Matematik ve mühendislik uygulama alanlarının yanında ekonomi ve olasilik teorisinde de kullanılmıştır. Son yıllarda Newtonyen olmayan kalkülüs birçok yazar tarafından çalış1lmıştır. Bunlardan bazıları; Çakmak ve Başar Newtonyen olmayan dizi uzayları (Çakmak ve Başar, 2012), Bashirov ve Rıza çarpımsal kompleks kalkülüs (Bashirov ve Riza, 2011), Florak ve Assen Biomedikal görüntü analizi (Florak ve Assen, 2012), Duyar ve Oğur Newtonyen olmayan reel sayıların topolojisi (Duyar ve Oğur, 2017), Duyar ve Sağır Newtonyen olmayan açık kümelerin Lebesgue ölçüsü (Duyar ve Sağır, 2017) üzerinde çalışmalar yapmışlardır.

Bu çalışmada (Duyar ve Sağır, 2017) ve (Oğur ve Demir, 2019) çalışmalarından faydalanarak, Newtonyen olmayan iç ölçü, dış ölçü ve Lebesgue ölçüsü tanımlarının yanında ilgili temel teoremler verildi.

\section{2. Ön Bilgiler}

$\mathrm{Bu}$ bölümde kullanılacak bazı temel tanım ve teoremler verilecektir.

Tanım 2.1. Tanım kümesi $\mathbb{R}$ ve görüntü kümesi $A \subset \mathbb{R}$ olan birebir fonksiyona üreteç denir. Görüntü kümesi A olan bir $\gamma$ üreteci düşünülsün. $\forall p, q \in A$ için verilen işlemler ve sıralama bağıntısıyla birlikte

$\gamma$-toplam

$p \dot{+} q=\gamma\left\{\gamma^{-1}(p)+\gamma^{-1}(q)\right\}$

$\gamma-$ çıkarma

$p \circ q=\gamma\left\{\gamma^{-1}(p)-\gamma^{-1}(q)\right\}$

$\gamma$ - çarpma

$p \dot{\times} q=\gamma\left\{\gamma^{-1}(p) \times \gamma^{-1}(q)\right\}$

$\gamma$-bölme $\quad(q \neq \dot{0})$

$p \dot{/} q=\gamma\left\{\gamma^{-1}(p) / \gamma^{-1}(q)\right\}$

$\gamma$-siralama

$p \dot{\leq} q \Leftrightarrow \gamma^{-1}(p) \leq \gamma^{-1}(q)$

$\alpha$-aritmetik olarak ifade edilir. $\mathbb{R}(N)=\{\gamma(p): p \in \mathbb{R}\} \quad$ kümesine Newtonyen olmayan reel sayı kümesi denir (Grossman ve Katz, 1967).

Tanım 2.2. $\mathbb{R}(\mathbb{N})^{\prime}$ deki $(p, q)_{N} \quad \gamma-\operatorname{aç1k}$ aralığının ölçüsü

$m_{N}(p, q)_{N}=\gamma\left(m\left(\gamma^{-1}(p), \gamma^{-1}(q)\right)\right)$

şeklinde tanımlanır (Duyar ve Sağır, 2017).

Tanım 2.3. $\mathbb{R}(\mathbb{N})$ ' deki boştan farklı bir $\mathrm{F} \gamma-$ açık kümesinin Newtonyen olmayan ölçüsü, bileşen aralıklarının Newtonyen olmayan ölçülerinin toplamıdır: yani

$m_{N}(F)={ }_{N} \sum_{k} m_{N}\left(\delta_{k}\right)$

Burada $\delta_{k}=\left(p_{k}, q_{k}\right)_{N}$ ile tanımlıdır (Duyar ve Sağır, 2017).

Tanım 2.4. $\mathbb{R}(N)$ 'de boştan farklı $\gamma-$ sınırlı, $\gamma$-kapalı $\mathrm{F}$ kümesinin ölçüsü

$m_{N} F=\gamma\left\{m\left(\gamma^{-1}(A), \gamma^{-1}(B)\right)-m\left(\gamma^{-1}\left(C_{S}^{F}\right)\right)\right\}$

şeklinde tanımlanır. Burada $\mathrm{S}=[\mathrm{A}, \mathrm{B}]_{\mathrm{N}}, \quad F$ kümesini içeren en küçük $\gamma$ - kapalı aralık ve $\mathrm{C}_{\mathrm{S}}^{\mathrm{F}}=\mathrm{S}-\mathrm{F} \quad \gamma-$ açıktır (Oğur ve Demir, 2019).

\section{Araştırma bulguları}

Tanım 3.1. Boştan farklı $\gamma-$ sinırlı bir $E$ kümesinin Newtonyen olmayan dış ölçüsü, $E$ kümesini içeren tüm tüm $\gamma-$ sinırlı, $\gamma-$ açık kümelerin ölçülerinin en büyük alt sınırıdır; yani $\mathrm{m}_{\mathrm{N}}^{*} \mathrm{E}={ }^{\gamma} \inf _{\mathrm{E} \in \mathrm{G}}\left\{\mathrm{m}_{\mathrm{N}} \mathrm{G}\right\}$

ile tanımlanır.

Tanım 3.2. Boştan farklı, $\gamma-$ sinırlı bir $E$ kümesinin Newtonyen olmayan iç ölçüsü, $E$ kümesinin içerdiği tüm $\gamma-$ kapalı kümelerin ölçülerinin en küçük üst sınırıdır. Yani

$\mathrm{m}_{* \mathrm{~N}} \mathrm{E}={ }_{\mathrm{F} \in \mathrm{E}}^{\gamma} \sup _{\mathrm{N}}\left\{\mathrm{m}_{\mathrm{N}} \mathrm{F}\right\}$

ile tanımlanır.

Teorem 3.3. $G, \gamma-$ sinırlı, $\gamma$ - açık küme ise,

$\mathrm{m}_{\mathrm{N}}^{*} \mathrm{G}=\mathrm{m}_{*_{\mathrm{N}}} \mathrm{G}=\mathrm{m}_{\mathrm{N}} \mathrm{G}$

sağlanır.

İspat. $E, G$ kümesini içeren bir $\gamma$ - açık küme ve $F, G$ kümesinin $\gamma$ - kapalı bir alt kümesi olsun. 
Böylece

$$
\begin{aligned}
\mathrm{m}_{\mathrm{N}}^{*} \mathrm{G} & ={ }^{\gamma} \inf _{\mathrm{G} \subset \mathrm{E}}\left\{\mathrm{m}_{\mathrm{N}} \mathrm{E}\right\} \\
& =\gamma\left\{\inf _{\gamma^{-1}(\mathrm{G}) \subset \gamma^{-1}(\mathrm{E})} \gamma^{-1}\left\{\mathrm{~m}_{\mathrm{N}} \mathrm{E}\right\}\right\} \\
& =\gamma\left\{\inf _{\gamma^{-1}(\mathrm{G}) \subset \gamma^{-1}(\mathrm{E})} \gamma^{-1}\left(\gamma\left(\mathrm{m}\left(\gamma^{-1}(\mathrm{E})\right)\right)\right)\right\} \\
& =\gamma\left\{\inf _{\gamma^{-1}(\mathrm{G}) \subset \gamma^{-1}(\mathrm{E})} \mathrm{m}\left(\gamma^{-1}(\mathrm{E})\right)\right\} \\
& =\gamma\left\{\mathrm{m}\left(\gamma^{-1}(\mathrm{G})\right)\right\} \\
& =\mathrm{m}_{\mathrm{N}} \mathrm{G}
\end{aligned}
$$

ve

$$
\begin{aligned}
\mathrm{m}_{* \mathrm{~N}} \mathrm{G} & ={ }^{\gamma} \sup _{\mathrm{F} \in \mathrm{G}}\left\{\mathrm{m}_{\mathrm{N}} \mathrm{F}\right\} \\
& =\gamma\left\{\sup _{\gamma^{-1}(\mathrm{~F}) \subset \gamma^{-1}(\mathrm{G})} \gamma^{-1}\left\{\mathrm{~m}_{\mathrm{N}} \mathrm{F}\right\}\right\} \\
& =\gamma\left\{\sup _{\gamma^{-1}(\mathrm{~F}) \subset \gamma^{-1}(\mathrm{G})} \gamma^{-1}\left(\gamma\left(\mathrm{m}\left(\gamma^{-1}(\mathrm{~F})\right)\right)\right)\right\} \\
& =\gamma\left\{\sup _{\gamma^{-1}(\mathrm{~F}) \gamma^{-1}(\mathrm{G})} \mathrm{m}\left(\gamma^{-1}(\mathrm{~F})\right)\right\} \\
& =\gamma\left(\mathrm{m}\left(\gamma^{-1}(\mathrm{G})\right)\right) \\
& =\mathrm{m}_{\mathrm{N}} \mathrm{G}
\end{aligned}
$$

olduğundan ispat tamamlanır.

Teorem 3.4. $F \gamma-$ sınırlı, $\gamma$ - kapalı küme ise, $m_{N}^{*} F=m_{* N} F=m_{N} F$

eşitliği sağlanır.

İspat. $E, F \gamma$ - kapalı kümesini içeren $\gamma$ - açık bir küme, $G$ kümesi de $F$ kümesinin $\gamma$ - kapalı bir altkümesi olsun. Bu durumda

$$
\begin{aligned}
\mathrm{m}_{\mathrm{N}}^{*} \mathrm{~F} & ={ }^{\gamma} \inf _{\mathrm{F} \subset \mathrm{E}}\left\{\mathrm{m}_{\mathrm{N}} \mathrm{E}\right\} \\
& =\gamma\left\{\inf _{\gamma^{-1}(\mathrm{~F}) \subset \gamma^{-1}(\mathrm{E})} \gamma^{-1}\left\{\mathrm{~m}_{\mathrm{N}} \mathrm{F}\right\}\right\} \\
& =\gamma\left\{\inf _{\gamma^{-1}(\mathrm{~F}) \subset \gamma^{-1}(\mathrm{E})} \gamma^{-1}\left(\alpha\left(\mathrm{m}\left(\gamma^{-1}(\mathrm{E})\right)\right)\right)\right\} \\
& =\gamma\left\{\inf _{\gamma^{-1}(\mathrm{~F}) \subset \gamma^{-1}(\mathrm{E})} \mathrm{m}\left(\gamma^{-1}(\mathrm{E})\right)\right\} \\
& =\gamma\left\{\mathrm{m}\left(\gamma^{-1}(\mathrm{~F})\right)\right\} \\
& =\mathrm{m}_{\mathrm{N}} \mathrm{F}
\end{aligned}
$$

olur. Benzer şekilde

$$
\begin{aligned}
\mathrm{m}_{* \mathrm{~N}} \mathrm{~F} & ={ }^{\gamma} \sup _{\mathrm{G} \subset \mathrm{F}}\left\{\mathrm{m}_{\mathrm{N}} \mathrm{G}\right\} \\
& =\gamma\left\{\sup _{\gamma^{-1}(\mathrm{G}) \subset \gamma^{-1}(\mathrm{~F})} \gamma^{-1}\left\{\mathrm{~m}_{\mathrm{N}} \mathrm{G}\right\}\right\}
\end{aligned}
$$

$$
\begin{aligned}
& =\gamma\left\{\sup _{\gamma^{-1}(\mathrm{G}) \subset \gamma^{-1}(\mathrm{~F})} \gamma^{-1}\left(\gamma\left(\mathrm{m}\left(\gamma^{-1}(\mathrm{G})\right)\right)\right)\right\} \\
& =\gamma\left\{\sup _{\gamma^{-1}(\mathrm{G}) \subset \gamma^{-1}(\mathrm{~F})} \mathrm{m}\left(\gamma^{-1}(\mathrm{G})\right)\right\} \\
& =\gamma\left(\mathrm{m}\left(\gamma^{-1}(\mathrm{~F})\right)\right) \\
& =\mathrm{m}_{\mathrm{N}} \mathrm{F}
\end{aligned}
$$

bulunur.

Teorem 3.5. Her $\gamma-\operatorname{sinırlı~} E$ kümesi için

$\mathrm{m}_{{ }_{N}} \mathrm{E} \dot{\leq} \mathrm{m}_{\mathrm{N}}{ }_{\mathrm{N}} \mathrm{E}$

eşitsizliği geçerlidir.

İspat. $G, E$ kümesini içeren $\gamma$ - açık küme, $F$ kümesi de $E$ kümesinin $\gamma$ - kapalı bir alt kümesi olsun. Bu durumda

$$
\begin{aligned}
\mathrm{m}_{*_{\mathrm{N}}} \mathrm{E} & =\sup _{\mathrm{F} \subset \mathrm{E}}\left\{\mathrm{m}_{\mathrm{N}} \mathrm{F}\right\} \\
& =\gamma\left\{\sup _{\gamma^{-1}(\mathrm{~F}) \subset \gamma^{-1}(\mathrm{E})} \gamma^{-1}\left\{\mathrm{~m}_{\mathrm{N}} \mathrm{F}\right\}\right\} \\
& =\gamma\left\{\sup _{\gamma^{-1}(\mathrm{~F}) \subset \gamma^{-1}(\mathrm{E})} \gamma^{-1}\left(\gamma\left(\mathrm{m}\left(\gamma^{-1}(\mathrm{~F})\right)\right)\right)\right\} \\
& =\gamma\left\{\sup _{\gamma^{-1}(\mathrm{~F}) \subset \gamma^{-1}(\mathrm{E})} \mathrm{m}\left(\gamma^{-1}(\mathrm{~F})\right)\right\} \\
& \dot{\leq} \gamma\left\{\inf _{\gamma^{-1}(\mathrm{E}) \subset \gamma^{-1}(\mathrm{G})} \mathrm{m}\left(\gamma^{-1}(\mathrm{G})\right)\right\} \\
& =\gamma\left\{\inf _{\gamma^{-1}(\mathrm{E}) \subset \gamma^{-1}(\mathrm{G})} \gamma^{-1}\left(\gamma\left(\mathrm{m}\left(\gamma^{-1}(\mathrm{G})\right)\right)\right)\right\} \\
& =\gamma\left\{\inf _{\gamma^{-1}(\mathrm{E}) \subset \gamma^{-1}(\mathrm{G})} \gamma^{-1}\left\{\mathrm{~m}_{\mathrm{N}} \mathrm{G}\right\}\right\} \\
& ={ }^{\gamma} \inf _{\mathrm{E} \subset \mathrm{G}}\left\{\mathrm{m}_{\mathrm{N}} \mathrm{G}\right\} \\
& =\mathrm{m}^{*}{ }_{\mathrm{N}} \mathrm{E} \text { elde edilir. }
\end{aligned}
$$

Teorem 3.6. $A$ ve $B \quad \gamma-$ sinırlı kümeler olsun. $A \subset B$ ise

$m_{*_{N}} A \dot{\leq} m_{*_{N}} B$ ve $\mathrm{m}^{*} \mathrm{~A} \dot{\leq} \mathrm{m}^{*}{ }_{\mathrm{N}} \mathrm{B}$

eşitsizlikleri sağlanır.

İspat. $E, A$ kümesini içeren $\gamma$ - açık küme ve $G$ kümesi de $A$ kümesinin $\gamma$ - kapalı bir alt kümesi olsun. Böylece

$$
\begin{aligned}
\mathrm{m}_{*_{\mathrm{N}}} \mathrm{A} & ={ }^{\gamma} \sup _{G \subset \mathrm{A}}\left\{\mathrm{m}_{\mathrm{N}} G\right\} \\
& =\gamma\left\{\sup _{\gamma^{-1}(G) \subset \gamma^{-1}(\mathrm{~A})} \gamma^{-1}\left\{\mathrm{~m}_{\mathrm{N}} G\right\}\right\} \\
& =\gamma\left\{\sup _{\gamma^{-1}(G) \subset \gamma^{-1}(\mathrm{~A})} \gamma^{-1}\left(\gamma\left(\mathrm{m}\left(\gamma^{-1}(G)\right)\right)\right)\right\}
\end{aligned}
$$




$$
\begin{aligned}
& =\gamma\left\{\sup _{\gamma^{-1}(G) \subset \gamma^{-1}(\mathrm{~A})} \mathrm{m}\left(\gamma^{-1}(G)\right)\right\} \\
& \dot{\leq} \gamma\left\{\sup _{\gamma^{-1}(\mathrm{~L}) \subset \gamma^{-1}(\mathrm{~B})} \mathrm{m}\left(\gamma^{-1}(\mathrm{~L})\right)\right\} \\
& =\gamma\left\{\sup _{\gamma^{-1}(\mathrm{~L}) \subset \gamma^{-1}(\mathrm{~B})} \gamma^{-1}\left(\gamma\left(\mathrm{m}\left(\gamma^{-1}(\mathrm{~L})\right)\right)\right)\right\} \\
& =\gamma\left\{\sup _{\gamma^{-1}(\mathrm{~L}) \subset \gamma^{-1}(\mathrm{~B})} \gamma^{-1}\left\{\mathrm{~m}_{\mathrm{N}} \mathrm{L}\right\}\right\} \\
& =\gamma \sup _{\left.\mathrm{Sum}_{\mathrm{N}} \mathrm{L}\right\}}\left\{\mathrm{m}_{\mathrm{L} \subset \mathrm{B}}\right. \\
& =\mathrm{m}_{* \mathrm{~N}} \mathrm{~B}
\end{aligned}
$$

elde edilir. Diğer eşitsizlikte benzer şekilde elde edilebilir.

Teorem 3.7. $\gamma-\operatorname{sinırl}$ bir $E$ kümesi sonlu ya da sayılabilir sonsuz $E_{k}$ kümelerinin birleşimi, yani $E=\bigcup_{\mathrm{k}=1}^{\infty} E_{\mathrm{k}}$, olsun. Bu durumda

$m{ }_{\mathrm{N}} E \dot{\leq}_{\mathrm{N}} \sum_{\mathrm{k}=1}^{\infty} m^{*}{ }_{\mathrm{N}} E_{\mathrm{k}}$

eşitsizliği sağlanır.

İspat. $\sum_{\mathrm{N}} \sum_{\mathrm{k}=1}^{\infty} \mathrm{m}_{\mathrm{N}}^{*} \mathrm{E}_{\mathrm{k}} \quad \gamma-$ rraksak ise ispat açıktır. $\mathrm{Bu}$ serinin $\gamma$ - yakınsak olduğunu kabul edelim. $G, E$ kümesini içeren $\gamma$ - açık küme ve $G_{\mathrm{k}}$ kümeleri de $E_{k}$ kümelerini içeren $\gamma-$ açık kümeler olsun. Buradan $\mathrm{m}_{\mathrm{N}}^{*} \mathrm{E}={ }^{\gamma} \inf _{\mathrm{E} \subset \mathrm{G}}\left\{\mathrm{m}_{\mathrm{N}} \mathrm{G}\right\}$

$$
\begin{aligned}
& =\gamma\left\{\inf _{\gamma^{-1}(\mathrm{E}) \subset \gamma^{-1}(\mathrm{G})} \gamma^{-1}\left\{\mathrm{~m}_{\mathrm{N}} \mathrm{G}\right\}\right\} \\
& =\gamma\left\{\inf _{\gamma^{-1}(\mathrm{E}) \subset \gamma^{-1}(\mathrm{G})} \gamma^{-1}\left(\gamma\left(\mathrm{m}\left(\gamma^{-1}(\mathrm{G})\right)\right)\right)\right\} \\
& =\gamma\left\{\inf _{\gamma^{-1}(\mathrm{E}) \subset \gamma^{-1}(\mathrm{G})} \mathrm{m}\left(\gamma^{-1}(\mathrm{G})\right)\right\} \\
& \dot{\leq} \gamma\left\{\sum_{\mathrm{k}=1}^{\infty} \inf _{\gamma^{-1}\left(\mathrm{E}_{\mathrm{k}}\right) \subset \gamma^{-1}\left(\mathrm{G}_{\mathrm{k}}\right)} \mathrm{m}\left(\gamma^{-1}\left(\mathrm{G}_{\mathrm{k}}\right)\right)\right\} \\
& =\gamma\left\{\sum_{\mathrm{k}=1}^{\infty} \gamma^{-1}\left(\gamma\left(\inf _{\gamma^{-1}\left(\mathrm{E}_{\mathrm{k}}\right) \subset \gamma^{-1}\left(\mathrm{G}_{\mathrm{k}}\right)} \mathrm{m}\left(\gamma^{-1}\left(\mathrm{G}_{\mathrm{k}}\right)\right)\right)\right)\right\} \\
& =\sum_{\mathrm{N}} \sum_{\mathrm{k}=1}^{\infty} \gamma\left(\inf _{\gamma^{-1}\left(\mathrm{E}_{\mathrm{k}}\right) \subset \gamma^{-1}\left(\mathrm{G}_{\mathrm{k}}\right)}\left(\mathrm{m}\left(\gamma^{-1}\left(\mathrm{G}_{\mathrm{k}}\right)\right)\right)\right) \\
& =\sum_{\mathrm{N}} \sum_{\mathrm{k}=1}^{\infty} \gamma\left(\inf _{\gamma^{-1}\left(\mathrm{E}_{\mathrm{k}}\right) \subset \gamma^{-1}\left(\mathrm{G}_{\mathrm{k}}\right)} \gamma^{-1}\left(\gamma\left(\mathrm{m}\left(\gamma^{-1}\left(\mathrm{G}_{\mathrm{k}}\right)\right)\right)\right)\right) \\
& =\sum_{\mathrm{N}} \sum_{\mathrm{k}=1}^{\infty} \inf _{\mathrm{E}_{\mathrm{k}}} \operatorname{Gi}_{\mathrm{k}}\left(\mathrm{m}\left(\gamma^{-1}\left(\mathrm{G}_{\mathrm{k}}\right)\right)\right)
\end{aligned}
$$

$$
={ }_{\mathrm{N}} \sum_{\mathrm{k}=1}^{\infty} \mathrm{m}_{\mathrm{N}}^{*} \mathrm{E}_{\mathrm{k}} \quad \text { elde edilir. }
$$

Teorem 3.8. $\gamma-$ sinırlı bir $E$ kümesi sonlu ya da sayılabilir sonsuz sayıdaki ikişer ikişer ayrık $E_{k}$ kümelerinin birleşimi yani

$$
E=\bigcup_{\mathrm{k}} E_{\mathrm{k}} \quad\left(E_{\mathrm{k}} \cap E_{\mathrm{k}^{\prime}}=\varnothing \quad \mathrm{k} \neq \mathrm{k}^{\prime}\right)
$$

olsun. Bu durumda

$$
m_{*_{\mathrm{N}}} E \dot{\geq}_{\mathrm{N}} \sum_{\mathrm{k}=1}^{\infty} m_{*_{\mathrm{N}}} E_{\mathrm{k}}
$$

eşitsizliği sağlanır.

İspat. $G=\bigcup_{k} G_{k}, E$ kümesinde kapsanan bir $\gamma-$ kapalı küme ve $G_{k}$ kümeleri de $E_{k}$ kümelerinin $\gamma-$ kapalı altkümeleri olsunlar. Buradan

$$
\begin{aligned}
& \mathrm{m}_{*_{\mathrm{N}}} \mathrm{E}={ }^{\gamma} \sup _{\mathrm{G} \subset \mathrm{E}}\left\{\mathrm{m}_{\mathrm{N}} \mathrm{G}\right\} \\
& =\gamma\left\{\sup _{\gamma^{-1}(\mathrm{G}) \in \gamma^{-1}(\mathrm{E})} \gamma^{-1}\left\{\mathrm{~m}_{\mathrm{N}} \mathrm{G}\right\}\right\} \\
& =\gamma\left\{\sup _{\gamma^{-1}(\mathrm{G}) \subset \gamma^{-1}(\mathrm{E})} \gamma^{-1}\left(\gamma\left(\mathrm{m}\left(\gamma^{-1}(\mathrm{G})\right)\right)\right)\right\} \\
& =\gamma\left\{\sup _{\gamma^{-1}(\mathrm{G}) \subset \gamma^{-1}(\mathrm{E})} \mathrm{m}\left(\gamma^{-1}(\mathrm{G})\right)\right\} \\
& \dot{\geq} \gamma\left\{\sum_{\mathrm{k}=1}^{\infty} \sup _{\gamma^{-1}\left(\mathrm{G}_{\mathrm{k}}\right) \subset \gamma^{-1}\left(\mathrm{E}_{\mathrm{k}}\right)} \mathrm{m}\left(\gamma^{-1}\left(\mathrm{G}_{\mathrm{k}}\right)\right)\right\} \\
& =\gamma\left\{\sum_{\mathrm{k}=1}^{\infty} \gamma^{-1}\left(\gamma\left(\sup _{\gamma^{-1}\left(\mathrm{G}_{\mathrm{k}}\right) \subset \gamma^{-1}\left(\mathrm{E}_{\mathrm{k}}\right)} \mathrm{m}\left(\gamma^{-1}\left(\mathrm{G}_{\mathrm{k}}\right)\right)\right)\right)\right\} \\
& =\mathrm{N}_{\mathrm{N}} \sum_{\mathrm{k}=1}^{\infty} \gamma\left(\sup _{\gamma^{-1}\left(\mathrm{G}_{\mathrm{k}}\right) \subset \gamma^{-1}\left(\mathrm{E}_{\mathrm{k}}\right)} \mathrm{m}\left(\gamma^{-1}\left(\mathrm{G}_{\mathrm{k}}\right)\right)\right) \\
& ={ }_{\mathrm{N}} \sum_{\mathrm{k}=1}^{\infty} \gamma\left(\sup _{\gamma^{-1}\left(\mathrm{G}_{\mathrm{k}}\right) \in \gamma^{-1}\left(\mathrm{E}_{\mathrm{k}}\right)} \gamma^{-1}\left(\gamma\left(\mathrm{m}\left(\gamma^{-1}\left(\mathrm{G}_{\mathrm{k}}\right)\right)\right)\right)\right) \\
& ={ }_{\mathrm{N}} \sum_{\mathrm{k}=1}^{\infty}\left(\sup _{\mathrm{G}_{\mathrm{k}} \in \mathrm{E}_{\mathrm{k}}} \gamma\left(\mathrm{m}\left(\gamma^{-1}\left(\mathrm{G}_{\mathrm{k}}\right)\right)\right)\right)
\end{aligned}
$$

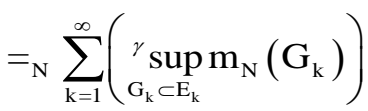

$$
\begin{aligned}
& ={ }_{\mathrm{N}} \sum_{\mathrm{k}=1}^{\infty} \mathrm{m}_{{ }_{\mathrm{N}}} \mathrm{E}_{\mathrm{k}}
\end{aligned}
$$

elde edilir.

Teorem 3.9. $\gamma-$ sinırlı bir $E$ kümesi verilsin. Eğer $\Delta, E$ kümesini içeren $\gamma$-açık bir aralık ise, $\mathrm{m}^{*}{ }_{\mathrm{N}} \mathrm{E}+\mathrm{m}_{*_{\mathrm{N}}}\left[\mathrm{C}_{\Delta}^{\mathrm{E}}\right]=\mathrm{m}_{\mathrm{N}} \Delta$

eşitliği sağlanır. 
İspat. $G, E$ kümesini içeren $\gamma$ - açık küme ve $K$ da $C_{\Delta}^{\mathrm{E}}$ kümesinin $\gamma-$ kapalı bir altkümesi olsun. $\mathrm{Bu}$ durumda

$$
\begin{aligned}
& \mathrm{m}^{*}{ }_{\mathrm{N}} \mathrm{E}+\mathrm{m}_{*_{\mathrm{N}}}\left[\mathrm{C}_{\Delta}^{\mathrm{E}}\right] \\
& =\gamma\left\{\gamma^{-1}\left(\mathrm{~m}^{*}{ }_{\mathrm{N}} \mathrm{E}\right)+\gamma^{-1}\left(\mathrm{~m}_{*_{\mathrm{N}}}\left[\mathrm{C}_{\Delta}^{\mathrm{E}}\right]\right)\right\} \\
& =\gamma\left\{\gamma^{-1}\left(\inf _{\mathrm{E} \subset \mathrm{G}}\left(\mathrm{m}_{\mathrm{N}} \mathrm{G}\right)\right)+\gamma^{-1}\left({ }_{\sup _{\mathrm{K} \subset \mathrm{C}_{\Delta}^{\mathrm{E}}}}\left(\mathrm{m}_{\mathrm{N}} \mathrm{K}\right)\right)\right\} \\
& =\gamma\left\{\gamma^{-1}\left(\gamma\left(\inf _{\gamma^{-1}(\mathrm{E}) \in \gamma^{-1}(\mathrm{G})} \gamma^{-1}\left(\mathrm{~m}_{\mathrm{N}} \mathrm{G}\right)\right)\right)+\gamma^{-1}\left(\gamma\left(\sup _{\gamma^{-1}(\mathrm{~K}) \subset \gamma^{-1}\left(\mathrm{E}_{\mathrm{N}}^{\mathrm{E}}\right)} \gamma^{-1}\left(\mathrm{~m}_{\mathrm{N}} \mathrm{K}\right)\right)\right)\right\} \\
& =\gamma\left\{\inf _{\gamma^{-1}(\mathrm{E}) \subset \gamma^{-1}(\mathrm{G})} \gamma^{-1}\left(\mathrm{~m}_{\mathrm{N}} \mathrm{G}\right)+\sup _{\gamma^{-1}(\mathrm{~K}) \subset \gamma^{-1}\left(\mathrm{C}_{\Delta}^{\mathrm{E}}\right)} \gamma^{-1}\left(\mathrm{~m}_{\mathrm{N}} \mathrm{K}\right)\right\} \\
& =\gamma\left\{\inf _{\gamma^{-1}(\mathrm{E}) \subset \gamma^{-1}(\mathrm{G})} \gamma^{-1}\left(\gamma\left(\mathrm{m}\left(\gamma^{-1}(\mathrm{G})\right)\right)\right)+\sup _{\gamma^{-1}(\mathrm{~K}) \subset \gamma^{-1}\left(\mathrm{C}_{\mathrm{E}}^{\mathrm{E}}\right)} \gamma^{-1}\left(\gamma\left(\mathrm{m}\left(\gamma^{-1}(\mathrm{~K})\right)\right)\right)\right\} \\
& =\gamma\left\{\inf _{\gamma^{-1}(\mathrm{E}) \subset \gamma^{-1}(\mathrm{G})} \mathrm{m}\left(\gamma^{-1}(\mathrm{G})\right)+\sup _{\gamma^{-1}(\mathrm{~K}) \subset \gamma^{-1}\left(\mathrm{C}_{\Delta}^{\mathrm{E}}\right)} \mathrm{m}\left(\gamma^{-1}(\mathrm{~K})\right)\right\} \\
& =\gamma\left\{\mathrm{m}^{*}\left(\gamma^{-1}(\mathrm{E})\right)+\mathrm{m}_{*}\left(\gamma^{-1}\left(\mathrm{C}_{\Delta}^{\mathrm{E}}\right)\right)\right\} \\
& =\gamma\left\{\mathrm{m}\left(\gamma^{-1}(\Delta)\right)\right\} \\
& =\mathrm{m}_{\mathrm{N}} \Delta \text {. }
\end{aligned}
$$

elde edilir.

Tanım 3.10. $\gamma-$ sinırlı bir $E$ kümesinin Newtonyen olmayan iç ve dış ölçüleri eşit ise, $E$ kümesine Newtonyen olmayan Lebesgue ölçülebilir küme ya da kısaca $\gamma$ - ölçülebilir küme denir.

$G, E$ kümesini içeren $\alpha$-açık küme ve $F$ kümesi de $E$ kümesinin $\alpha$-kapalı bir altkümesi olsun. Bu durumda $m^{*}{ }_{N} E={ }^{\gamma} \inf _{E \subset G}\left\{m_{N} G\right\}$

ve $\mathrm{m}_{*_{\mathrm{N}}} \mathrm{E}={ }_{\mathrm{F}}^{\gamma} \sup _{\mathrm{E}}\left\{\mathrm{m}_{\mathrm{N}} \mathrm{F}\right\}$

olur ve $\mathrm{m}_{*_{\mathrm{N}}} \mathrm{E}=\mathrm{m}^{*}{ }_{\mathrm{N}} \mathrm{E}$ olduğundan

$$
\begin{aligned}
& \mathrm{m}_{{ }_{N}} \mathrm{E}=\mathrm{m}^{*}{ }_{\mathrm{N}} \mathrm{E} \\
& \gamma \inf _{\mathrm{E} \subset \mathrm{G}}\left\{\mathrm{m}_{\mathrm{N}} \mathrm{G}\right\}={ }^{\gamma} \sup _{\mathrm{F} \subset \mathrm{E}}\left\{\mathrm{m}_{\mathrm{N}} \mathrm{F}\right\} \\
& \Rightarrow \gamma\left\{\inf _{\gamma^{-1}(\mathrm{E}) \gamma^{-1}(\mathrm{G})} \gamma^{-1}\left(\gamma\left(\mathrm{m}\left(\gamma^{-1}(\mathrm{G})\right)\right)\right)\right\}=\gamma\left\{\sup _{\gamma^{-1}(\mathrm{~F}) \subset \gamma^{-1}(\mathrm{E})} \gamma^{-1}\left(\gamma\left(\gamma^{-1}(\mathrm{~F})\right)\right)\right\} \\
& \Rightarrow \gamma\left\{\inf _{\gamma^{-1}(\mathrm{E}) \subset \gamma^{-1}(\mathrm{G})} \mathrm{m}\left(\gamma^{-1}(\mathrm{G})\right)\right\}=\gamma\left\{\sup _{\gamma^{-1}(\mathrm{~F}) \subset \gamma^{-1}(\mathrm{E})} \mathrm{m}\left(\gamma^{-1}(\mathrm{~F})\right)\right\}
\end{aligned}
$$

$$
\begin{aligned}
& \Rightarrow \gamma^{-1}\left(\gamma\left\{\inf _{\gamma^{-1}(\mathrm{E}) \subset \gamma^{-1}(\mathrm{G})} \mathrm{m}\left(\gamma^{-1}(\mathrm{G})\right)\right\}\right)=\gamma^{-1}\left(\gamma\left\{\sup _{\gamma^{-1}(\mathrm{~F}) \gamma^{-1}(\mathrm{E})} \mathrm{m}\left(\gamma^{-1}(\mathrm{~F})\right)\right\}\right) \\
& \Rightarrow \inf _{\gamma^{-1}(\mathrm{E}) \gamma^{-1}(\mathrm{G})} \mathrm{m}\left(\gamma^{-1}(\mathrm{G})\right)=\sup _{\gamma^{-1}(\mathrm{~F}) c \gamma^{-1}(\mathrm{E})} \mathrm{m}\left(\gamma^{-1}(\mathrm{~F})\right) \\
& \Rightarrow \mathrm{m}^{*}\left(\gamma^{-1}(\mathrm{E})\right)=\mathrm{m}_{*}\left(\gamma^{-1}(\mathrm{E})\right)
\end{aligned}
$$

yazılır. Bu tanıma göre $E$ kümesi $\gamma$ - ölçülebilir küme ise $\gamma^{-1}(\mathrm{E}), \mathbb{R}$ de ölçülebilirdir.

Teorem 3.11. $\gamma-$ sınırl1, $\gamma-$ açık kümeler $\gamma-$ ölçülebilirdir.

İspat. $F \quad \gamma-$ sinırlı, $\gamma-$ açık küme olsun. $\mathrm{Bu}$ durumda Teorem 2.3 gereğince $\mathrm{m}_{\mathrm{N}}{ }_{\mathrm{N}} \mathrm{F}=\mathrm{m}_{{ }_{\mathrm{N}}} \mathrm{F}$ olur. Bu durumda $F$ kümesi $\gamma$ - ölçülebilirdir.

Teorem 3.12. Her $\gamma-$ sinırlı, $\gamma-$ kapalı küme $\gamma-$ ölçülebilirdir.

İspat. $G \gamma-$ sinırlı, $\gamma-$ kapalı bir küme olsun. $\mathrm{Bu}$ durumda Teorem 2.4 gereğince $\mathrm{m}_{\mathrm{N}}^{*} \mathrm{G}=\mathrm{m}_{{ }_{\mathrm{N}} \mathrm{G}} \mathrm{G}$ yazılır. Dolayısıyla $G$ kümesi $\gamma$ - ölçülebilir bir kümedir.

Teorem 3.13. $\gamma-$ sinırlı bir $E$ kümesi verilsin. Eğer E kümesi ikişer ikişer ayrık $\gamma$ - ölçülebilir $E_{\mathrm{k}}$ kümelerin sonlu ya da sayılabilir sonsuz kümelerinin birleşimi şeklinde yazılabiliyorsa $\mathrm{E}$, $\gamma-$ ölçülebilirdir ve

$m_{\mathrm{N}} E={ }_{\mathrm{N}} \sum_{\mathrm{k}} m_{\mathrm{N}} E_{\mathrm{k}}$

eşitliği sağlanır.

İspat: $\quad E_{\mathrm{k}} \cap E_{\mathrm{k}^{\prime}}=\varnothing \quad \mathrm{k} \neq \mathrm{k}^{\prime}, \quad$ olmak üzere $E=\bigcup_{\mathrm{k}} E_{\mathrm{k}}$ şeklinde yazılsın. Bu durumda $m_{\mathrm{N}}{ }_{\mathrm{N}} E \dot{\leq}_{\mathrm{N}} \sum_{\mathrm{k}} m^{*}{ }_{\mathrm{N}} E_{\mathrm{k}}$ ve $m_{*_{\mathrm{N}}} E \dot{\geq}_{\mathrm{N}} \sum_{\mathrm{k}} m_{*_{\mathrm{N}}} E_{\mathrm{k}}$ yazılır. $E, \quad \gamma-$ sinırlı bir küme olduğundan $m_{* \mathrm{~N}} E \dot{\leq} m^{*}{ }_{\mathrm{N}} E$ dir.

$E_{\mathrm{k}}$ kümeleri $\alpha$ - ölçülebilir kümeler olduğundan ${ }_{\mathrm{N}} \sum_{\mathrm{k}} m_{\mathrm{N}} E_{\mathrm{k}}=\sum_{\mathrm{k}} m_{\mathrm{N}_{\mathrm{N}}} E_{\mathrm{k}} \dot{\leq} m_{{ }_{\mathrm{N}} \mathrm{N}} E \dot{\leq} m_{\mathrm{Na}}^{*} E \dot{\leq}_{\mathrm{N}} \sum_{\mathrm{k}} m_{\mathrm{N}}^{*} E_{\mathrm{k}}={ }_{\mathrm{N}} \sum_{\mathrm{k}} m_{\mathrm{N}} E_{\mathrm{k}}$ yazilır. Dolayısıyla $m_{*_{\mathrm{N}}} E=m^{*}{ }_{\mathrm{N}} E$ dir. $\mathrm{Bu}$ durumda E kümesi $\gamma$ - ölçülebilirdir ve

$$
m_{\mathrm{N}} E={ }_{\mathrm{N}} \sum_{\mathrm{k}} m_{\mathrm{N}} E_{\mathrm{k}}
$$

elde edilir. 
Teorem 3.14. Sonlu sayıda $\gamma-$ ölçülebilir kümenin birleşimi yine $\gamma$ - ölçülebilirdir.

İspat: $E_{k} \quad$ kümeleri $\quad \gamma-$ ölçülebilir kümeler olmak üzere $\mathrm{E}=\bigcup_{\mathrm{k}=1}^{\mathrm{n}} \mathrm{E}_{\mathrm{k}}$ olsun. $\mathrm{E}_{\mathrm{k}}$ kümeleri $\gamma-$ ölçülebilir olduğundan $\gamma^{-1}\left(\mathrm{E}_{\mathrm{k}}\right)$ kümeleri $\mathbb{R}$ de ölçülebilirdir. $\mathrm{Bu}$ durumda $\bigcup_{\mathrm{k}=1}^{\mathrm{n}} \gamma^{-1}\left(\mathrm{E}_{\mathrm{k}}\right)$ kümeleri de ölçülebilirdir. Buradan $\bigcup_{\mathrm{k}=1}^{\mathrm{n}} \gamma^{-1}\left(\mathrm{E}_{\mathrm{k}}\right)=\gamma^{-1}\left(\bigcup_{\mathrm{k}=1}^{\mathrm{n}} \mathrm{E}_{\mathrm{k}}\right)=\gamma^{-1}(\mathrm{E})$ yazılır. Böylece $\gamma^{-1}(\mathrm{E})$ ölçülebilirdir. $\mathrm{Bu}$ ise $E$ kümesinin $\gamma-$ ölçülebilir küme olduğunu gösterir.

Teorem 3.15. Sonlu sayıda $\gamma-$ ölçülebilir kümenin kesişimi yine $\gamma$ - ölçülebilir kümedir.

İspat. $\mathrm{E}_{\mathrm{k}}$ kümeleri $\gamma-$ ölçülebilir kümeler olmak üzere $\mathrm{E}=\bigcap_{\mathrm{k}=1}^{\mathrm{n}} \mathrm{E}_{\mathrm{k}}$ olsun. $\mathrm{E}_{\mathrm{k}}$ kümeleri $\gamma-$ ölçülebilir olduğundan $\quad \gamma^{-1}\left(\mathrm{E}_{\mathrm{k}}\right) \quad$ kümeleri ölçülebilirdir. Dolayısıyla $\bigcap_{\mathrm{k}=1}^{\mathrm{n}} \gamma^{-1}\left(\mathrm{E}_{\mathrm{k}}\right)$ kümesi de ölçülebilirdir.

Ayrica

$\bigcap_{\mathrm{k}=1}^{\mathrm{n}} \gamma^{-1}\left(\mathrm{E}_{\mathrm{k}}\right)=\gamma^{-1}\left(\bigcap_{\mathrm{k}=1}^{\mathrm{n}} \mathrm{E}_{\mathrm{k}}\right)=\gamma^{-1}(\mathrm{E})$

yazılabileceğinden $\quad \gamma^{-1}(\mathrm{E}) \quad$ ölçülebilirdir. Dolayısıyla $E$ kümesi $\gamma-$ ölçülebilir kümedir.

Teorem 3.16. İki $\gamma$ - ölçülebilir kümenin farkları da $\gamma$ - ölçülebilir bir kümedir.

İspat. $\mathrm{E}_{1}$ ve $\mathrm{E}_{2}$ kümeleri $\gamma-$ ölçülebilir kümeler olmak üzere $\mathrm{E}=\mathrm{E}_{1} \backslash \mathrm{E}_{2}$ olsun. $\mathrm{E}_{1}$ ve $\mathrm{E}_{2}$ kümeleri $\gamma$ - ölçülebilir olduğundan $\gamma^{-1}\left(\mathrm{E}_{1}\right)$ ve $\gamma^{-1}\left(\mathrm{E}_{2}\right)$ kümeleri de ölçülebilirdir. Dolayısıyla $\gamma^{-1}\left(\mathrm{E}_{1}\right) \backslash \gamma^{-1}\left(\mathrm{E}_{2}\right)$ kümesi de ölçülebilirdir. Böylece $\gamma^{-1}\left(\mathrm{E}_{1}\right) \backslash \gamma^{-1}\left(\mathrm{E}_{2}\right)=\gamma^{-1}\left(\mathrm{E}_{1} \backslash \mathrm{E}_{2}\right)=\gamma^{-1}(\mathrm{E})$

olduğundan $\gamma^{-1}(\mathrm{E})$ ölçülebilir bir kümedir. Buradan E kümesi $\gamma$ - ölçülebilirdir.

Teorem 3.17. $E_{1}$ ve $E_{2} \quad \gamma$-ölçülebilir kümeler olsun. Eğer $E_{2} \subset E_{1}$ ve $E=E_{1} \backslash E_{2}$ ise $m_{\mathrm{N}} E=m_{\mathrm{N}} E_{1} \div m_{\mathrm{N}} E_{2} \quad$ sağlanır.
İspat.: $E_{1}$ ve $E_{2} \quad \gamma$ - ölçülebilir olduğundan Teorem 2.16 gereğince $E_{1} \backslash E_{2}$ de $\gamma-$ ölçülebilirdir. $E=E_{1} \backslash E_{2}$ olduğundan $E_{1}=E \cup E_{2}$ yazilır. Teorem 2.13 gereğince

$m_{\mathrm{N}} E_{1}=m_{\mathrm{N}} E \dot{+} m_{\mathrm{N}} E_{2}$ olur.

\section{Kaynaklar}

Bashirov, A.E. ve Riza, M., 2011. On complex multiplicative differentiation. TWMS Journal of Applied and Engineering Mathematics, 1(1), 7585.

Çakmak, A. F. ve Başar F., 2012. Some new results on sequence spaces with respect to non-Newtonian calculus. Journal of Inequalities and Applications, 228, 1-12.

Duyar, C. ve Oğur, O., 2017. A note on topology of non-Newtonian real numbers. Journal of Mathematics, 13(6), 11-14.

Duyar, C., Sağır, B. ve Oğur, O., 2015. Some basic topological properties on non-Newtonian real line. British Journal of Mathematics and Computer Science, 9(4), 300-307.

Duyar, C. ve Sağır, B., 2017. Non-Newtonian comment of Lebesgue measure in real numbers. Journal of Mathematics, Article ID 6507013, 1-4.

Erdoğan, M., 2016. Newtonyen olmayan reel say1 serileri ve has olmayan integraller, Yüksek Lisans Tezi, Ondokuz Mayıs Üniversitesi, Fen Bilimleri Enstitüsü, Samsun.

Florak, L. ve Assen, H. V., 2012. Multiplicative calculus in biomedical image analysis. Journal of Mathematical Imaging and Vision, 42(1), 6475 .

Grossman, M. ve Katz, R., 1972. Non-Newtonian calculus, $1^{\text {st }}$ ed. Press, Piagen Cove Massuchusets.

Kadak, U., 2015. Newtonyen olmayan analiz ve çeşitli uygulamaları, Doktora Tezi, Gazi Üniversitesi Fen Bilimleri Enstitüsü, Ankara.

Natanson, I. P., 1964. Theory of function of a real variable, vol 1, Frederick Ungar Publishing Co., New York, NY, USA.

Oğur, O. ve Demir, S., 2019. On non-Newtonian measure for $\alpha$-closed sets. New Trends in Mathematical Sciences, 7(2), 202-207. 